\title{
Identity based Encryption with Cloud Revocation Authority
}

\author{
D. Vishnu Vardhan Reddy, K. Jaisharma, T. Devi
}

\begin{abstract}
Identity-based coding/encryption (IBE) is a public key encrypted system that take outs the strain of public key infrastructure (PKI) and certified administration in standard crypto public key settings. In this public key system is not used, the downside may be a crucial thing in IBE settings. Many IBE schemes are proposed relating to this issue. Recently, by embedding associate degree computation techniques has been into IBE, Li et al. proposed an Identity-based encryption theme along with a keyupdate cloud service supplier. Their theme has 2 things one is that the computation overhead and other is communication prices are more than previous IBE schemes. The defect is lack of quantify ability within the sense that the key-update cloud service supplier should keep a secret worth for every user. With this article, we have a tendency to propose a replacement rescindable IBE theme with a cloud revocation authority (CRA) to solve the problems of 2 short things. The work is drastically improved and also the cloud revocation authority holds a secret for all users. For security purpose, we have a tendency to show that the proposed theme is totally secure beneath the additive Diffie-Hellman key Exchange (DBDH) assumption. Finally, we have a tendency to extend the proposed Identity-based encryption theme to gift a CRA cloud revocation authority authentication theme with limited privileges for an oversized range of assorted cloud technique services.
\end{abstract}

Keywords: cipher text, Crypto algorithms, public key, private key, plain text, user personal files

\section{INTRODUCTION}

Identity-based coding/encryption (IBE) is a public key encrypted system that take outs the strain of public key infrastructure (PKI) and certified administration in standard crypto public key settings. This ID-PKS setting have users and third party (personal key generator (master key)). The (personal key generator is accountable to come up with every user personal key with exploitation of data like (Gmail address, phone number etc). so, for this system there is no need of public key infrastructure and certified administration within the cryptologic things beneath ID-PKS.

Revised Manuscript Received on December 12, 2019

D. Vishnu Vardhan Reddy, Department of Computer Science and Engineering, Saveetha School of Engineering, Saveetha Institute of Medical and Technical Sciences

K. Jaisharma, Department of Computer Science and Engineering, Saveetha School of Engineering, Saveetha Institute of Medical and Technical Sciences

T. Devi, Department of Computer Science and Engineering, Saveetha School of Engineering, Saveetha Institute of Medical and Technical Sciences
In this case, Id-based cryptography permits a sender (user) to inscribe data directly by employing a receiver valid id on the authentication of public key of user. Along with it, the user(receiver) uses a personal key related to the persons valid id to rewrite cipher data. Although a public key system has got to offer a user cloud revocable system. The analysis of the problem on the way to takeout compromised users in Associate to Identity-Public Key System setting. Many standard public key systems, certificate cloud revocation list (CRL) may be a well-known revocable approach. within the certificate cloud revocation list approach, if a person has got a public key along with valid certificate, then the person initial authenticates them so appearance up the certificate cloud revocation to confirm that the general public key system has not been cancelled. In this case, the process needs the help of Public Key System so it'll help the communication to boost the consummation, many economical revocable themes for standard public cryptographic key settings are for Public Key Infrastructure. Scientists conjointly listen to the revocable problem of ID-Public Key System settings. Many revocable IBE schemes are planned concerning the revocation mechanisms in ID-Public Key System settings. In 2003, two scientists have planned the primary sensible IBE theme from the wail pairing and steered a straightforward revoked methodology during which every unrevoked user will receive a brand-new personal key given by the PKG sporadically.

\section{LITERATURE REVIEW}

[1]S..GUNJAL et al suggested that many systems enable information to its cloud user if a cloud user contains a bound set of attributes. Presently, one technique to see such policies is to use associate degree access to the cloud server to keep up the user information and have authentication management for it. At some times, when one of the servers is keeping information is compromised, the safety of the user information is compromised. For obtaining access management, information security and getting computing results, the data house owners ought to keep id-based security to encode the keep information. During the delegation of knowledge on cloud, the cloud servers could also be tampered by the counterfeit cipher-text. Furthermore, the licensed users could also be cheated by retorting them that they're un authorized. Largely the encoding management access attribute policies are advanced. During this paper, we present Cipher-encrypted text Policy encoding for maintaining advanced access management over encrypted information with verifiable customizable authorization.

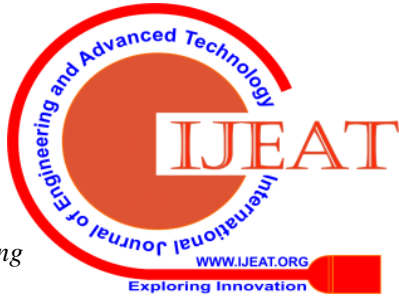


This plan provides information confidentiality to the encrypted information even though the server is full. Moreover, our method is extremely secured. [2]Sana Belgith et al proposed that Cloud-assisted IoT applications are gaining Associate in Nursing increasing interest, such IoT devices are deployed in several distributed environments to gather and source perceived information to remote servers for any process and sharing among users. On the one hand, in many applications, collected information are very sensitive and wish to be secured before outsourcing. Usually, the encrypted techniques are at $\{\mid$ the information $\}$ producer face to safeguard data from peoples as well as cloud supplier. On the opposite hand, sharing information among users needs fine grained access management mechanisms. to make sure each necessity, Attribute based mostly encoding (ABE) has been wide applied to make sure encrypted access management to outsourced information. Although, $\mathrm{ABE}$ ensures fine grained access management and information confidentiality, updates of used access policies when encryption Associate in outsourcing of knowledge remains an open challenge. during of key policy attribute based mostly encoding supporting economical access policy update that captures attributes addition to access policies.

\section{PROPOSED SYSTEM}

In our system we have a data provider, key authority(KA) [9],cloud and users.

\section{Data Provider}

The data supplier initial decides WHO can share the info and he can transfer the info with their identities. The info supplier uploads the info by encrypting it. Knowledge supplier will check for variety of uploads and variety of downloads of the info.

\section{Key Authority}

The key authority generates secret key when a user requests for data accessing

\section{Cloud Storage}

The knowledge uploaded by data supplier is hold on in cloud. The cloud allows the users to transfer by coming into the key. The cloud storage also will have a revocation list, if Associate in Nursing unauthorized/authority invalid user tries to access knowledge he/she is revoked. The revoked user isn't allowed to login once more.

\section{User}

The operate of the user is to request for secret key and accessing the information keep in cloud. The user decrypts the information by downloading it from cloud. If an unauthorized user tries to login it'll show a message, your underneath revocation. If the user is allowed and nevertheless revoked because of his/her expiration he/she may be unrevoked by checking the validity to access the information. To implement this, we tend to propose associate degree identity based mostly this paper, we have a tendency to style PU-ABE, a new variant

coding model employing a binary tree structure for storing identities and fundamental quantity functionalities of the users. Currently we tend to take a binary tree B, revocation list RL, current time revocation time and nodes as vi . Take 2 null sets X, Y cherish non-revoked and revoked users. If associate degree unauthorized/authority invalid shopper desires to access the shared knowledge he/she should use secret key. At the time of accessing cipher text, if the users current time is over the revocation time, he/she is marked as revoked and so prevented from additional access of shared knowledge. The non-revoked user's secret key's updated and that they will access the information victimization updated secret key that is provided by the key authority.

\section{SYSTEM ARCHITECTURE}

System architecture says that how plain text is converted into cipher text by the ide encryption. It also shows how the things will work diagrammatically.
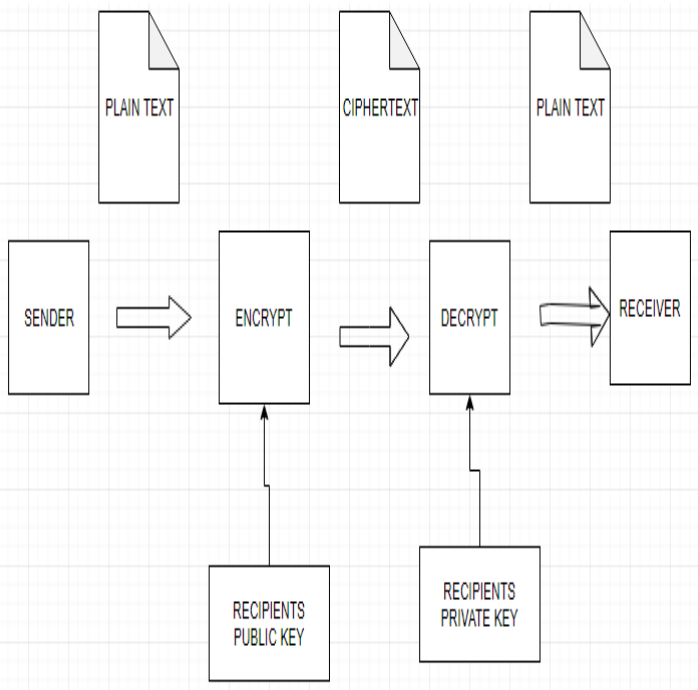

Fig. 1 Encryption and Decryption model

\section{RESULTS}

We have got the all the mails of users who wanted to transfer their data. And with the help of master key and personal data we will generate unique public keys. With that finally send data. The following comparison table shows the performance between the existing scheme Key-update cloud service providers with our proposed cloud revocation authority. The computational cost of consumes $4.3 \mathrm{~ms}$ using 2 keys for encryption and decryption. The time consumes for encryption is $0.43 \mathrm{~s}$ and to decrypt $0.18 \mathrm{~s}$. The cipher text size used for encryption is 148 bytes. So, it performs well compared to key-update cloud service provider scheme.

\section{Published By:}

Blue Eyes Intelligence Engineering

\& Sciences Publication 
Table. 1 Performance comparison between existing scheme and proposed scheme

\begin{tabular}{|l|l|l|}
\hline Comparison & Key-Update Cloud Service Provider (KU-CSP) & $\begin{array}{l}\text { Cloud Revocation Authority (IBE- } \\
\text { CRA) }\end{array}$ \\
\hline Computing time cost & $5.6(\mathrm{~ms})$ & $4.3(\mathrm{~ms})$ \\
\hline Number of keys & 1 & 2 \\
\hline Encryption computation & $0.643(\mathrm{~s})$ & $0.432(\mathrm{~s})$ \\
\hline Decryption computation & $0.26(\mathrm{~s})$ & $0.18(\mathrm{~s})$ \\
\hline Ciphertext size & 168 bytes & 148 bytes \\
\hline
\end{tabular}

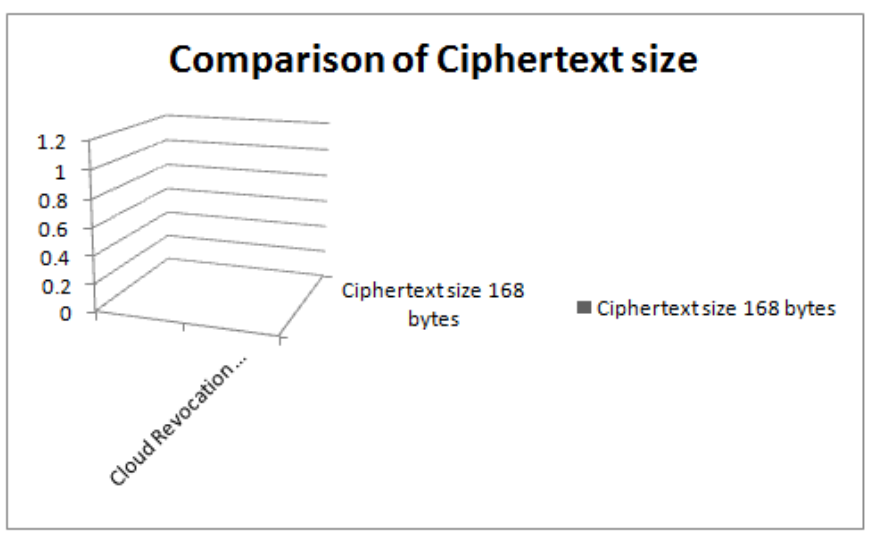

Fig. 2 Comparison chart between Ciphertext Size

\section{CONCLUSION}

The proposed System Revocable outsourcing IBE system is totally based on the CRA Authorization, In this system a revocation is performed by CRA for dealing with an impact of a PKG server, at that point the CRA Host creates an ace meter key products of the soil that to the client so the uprightness of the file organizer ought to be keep up, Whenever the recipient ask for record, Every fourth measurement another ace time key will be created for a specific client. Disseminated and layered approach is additionally extremely productive to determine the current system issues in IBE.

\section{REFERENCES}

1. K. Ren, C. Wang, and Q. Wang, - Security challenges for the public cloud, IEEE Internet Computing, vol. 16, pp. 69-73, Jan. 2012.

2. D. X. Song, D. Wagner, and A. Perrig, - Practical techniques for searches on encrypted data, in Security and Privacy, 2000. Sand P 2000. Proceedings. 2000 IEEE Symposium on, pp. 0-44, 2002.

3. E. J. Goh, - Secure indexes, Cryptology e Print Archive, http:// eprint.iacr.org/2003/216., 2003.

4. R. Curt mola, J. Garay, S. Kamara, and R. Ostrov sky, - Searchable symmetric encryption: improved definitions and efficient constructions, in ACM Conference on Computer and Communications Security, pp. 7988, 2006.

5. J. Li, Y. Shi, and Y. Zhang, - Searchable cipher text-policy attributebased encryption with revocation in cloud storage , International Journal of Communication Systems, vol. 30, no. 1, 2017.

6. Y. Miao, J. Ma, X. Liu, X. Li, Q. Jiang, and J. Zhang, -Attribute- based keyword search over hierarchical data in cloud computing IEEE Transactions on Services Computing, vol. PP, no. 99, pp. 1-1, 2017.

7. A. Swaminathan, Y. Mao, G. M. Su, H. Gou, A. L. Varna, S. He, M. Wu, and D. W. Oard, -Confidentiality-preserving rank-ordered search,\| in ACM Workshop on Storage Security and Survivability, Storagess 2007, Alexandria, Va, Usa, October, pp. 7-12, 2007.

8. Nadzir M., Hussain A., Mkpojiogu E.O.C., Faromiki J.O., Abdusalam E.M.A. (2019). The Effectiveness And Efficiency Of A GPS Route And Voice Navigation App. International Journal of Innovative Technology and Exploring Engineering. Vol 8. Issue 8. Page 425-427

9. C. Wang, N. Cao, K. Ren, and W. Lou, -Enabling secure and efficient ranked keyword search over outsourced cloud data, IEEE Transactions on Parallel and Distributed Systems, vol. 23, pp. 1467-1479, Aug.2012.

10. S. Zerr, D. Olmedilla, W. Nejdl, and W. Siberski, -Zerber $+\mathrm{r}$ : top- k retrieval from a confidential index, $\|$ in International Conference on Extending Data Base technology.

11. T. Padmapriya, S.V. Manikanthan, "LTE-A Intensified Voice Service Coder using TCP for Efficient Coding Speech", International Journal of Innovative Technology and Exploring Engineering, Vol. 8, issue 7s, 2019. https://www.ijitee.org/wp-

content/uploads/papers/v8i7s/G10630587S19.pdf 